\title{
Hearing improvement after vestibular schwannoma surgery in the era of the hearing preservation rule - case report and literature review
}

\author{
Zdenek Fik, Jan Lazak, Silvie Hruba, Zdenek Cada, Eduard Zverrna, Jan Betka
}

\begin{abstract}
Objectives. Hearing preservation after vestibular schwannoma (VS) surgery remains a surgical challenge. In some patients with preserved inner ear function, hearing improvement is achievable. As it is currently impossible to determine which patients will present this outcome, predictions must rely on previously published reports. Our case report describes a patient who experienced hearing improvement from an unuseful level to a useful one after vestibular schwannoma surgery.

Methods. Surgery was performed via suboccipital retrosigmoid approach. The patient underwent a basic audiovestibular protocol before and after the surgery - pure tone and speech audiometry, otoacoustic emissions, auditory brainstem responses, electronystagmography - together with a detailed questionnaire study. Usefulness of hearing was evaluated using the AAO-HNS guidelines, supplemented by a frequency of $4 \mathrm{kHz}$.

Results. Hearing was preserved and even improved from an unuseful level to a useful one. Based on the available literature, the most informative predictive factors for such a result seem to be: sudden sensorineural hearing loss prior to surgery, elicitable otoacoustic emissions and the origin from the superior vestibular nerve.

Conclusion. There are a limited number of studies on this topic and it is still impossible to regularly improve hearing in properly selected patients. Furthermore, the importance of postoperative hearing quality compared to other symptoms and complications remains debatable.
\end{abstract}

Key words: vestibular schwannoma, hearing improvement, retrosigmoid approach, quality of life

Received: February 20, 2021; Revised: September 3, 2021; Accepted: November 16, 2021; Available online: December 9, 2021 https://doi.org/10.5507/bp.2021.067

(c) 2022 The Authors; https://creativecommons.org/licenses/by/4.0/

Department of Otorhinolaryngology, Head and Neck Surgery, First Faculty of Medicine, Charles University in Prague and University Hospital Motol, V Uvalu 84, 150 06, Prague 5, Czech Republic

Corresponding author:Zdenek Fik, e-mail:zdenek.fik@fnmotol.cz

\section{INTRODUCTION}

The success of hearing preservation varies in the literature from $17 \%$ to $100 \%$ and depends on the size of the tumour, the choice of surgical approach and the surgeon's general attitude toward the issue ${ }^{1}$.

The mechanism of hearing loss in patients with VS is complex. In addition to compression of the auditory nerve by the tumour, restriction of the vascular supply of the inner ear and parasitism by the tumour (steal phenomenon), the influence of tumoural paracrine activity has been proposed as another responsible factor in recent years ${ }^{2}$.

The hearing damage caused during surgery stems from several factors which can either be positively or negatively influenced. These are direct damage of the auditory nerve by manipulation and damage of the vascular supply of the inner ear ${ }^{3}$. In addition, concerning vascularization of the inner ear, it is necessary to account for a typical property of cerebral vessels - the potential to vasospasm during irritation.

In general, the highest chances for hearing preservation are presented with tumours up to $15 \mathrm{~mm}$ in size, which can be defined as the "hearing preservation rule“ (PTA $<50 \mathrm{~dB}$, SDS $>50 \%$, largest dimension $<1.5 \mathrm{~cm}$ ).
This rule can be used to detect patients in whom it is desirable to try to preserve hearing $\mathrm{g}^{4,5}$.

Finally, the decision of whether to preserve hearing is seen in a completely different light when considering the possibility of hearing improvement after the surgery. A limited number of studies on this topic exist and we will present our own experience with such a result, together with possible predictive factors.

\section{LITERATURE SEARCH AND SELECTION}

A systematic search of the literature was completed using the PubMed/MEDLINE database for articles on hearing preservation in vestibular schwannoma surgery published till January December 2019, using the keywords "vestibular schwannoma," "acoustic neuroma/neurinoma," "hearing preservation," "hearing restoration," "hearing improvement,"in the syntax below, yielding 385 papers.

Pubmed Search Syntax

(("vestibular schwannoma") OR ("acoustic neurinoma") OR ("acoustic neurinoma")) AND (("hearing improvement") OR ("hearing preservation") OR ("hearing restoration”)) AND (surgery OR microsurgery) 
Table 1. Comparison of preoperative and postoperative audiometry.

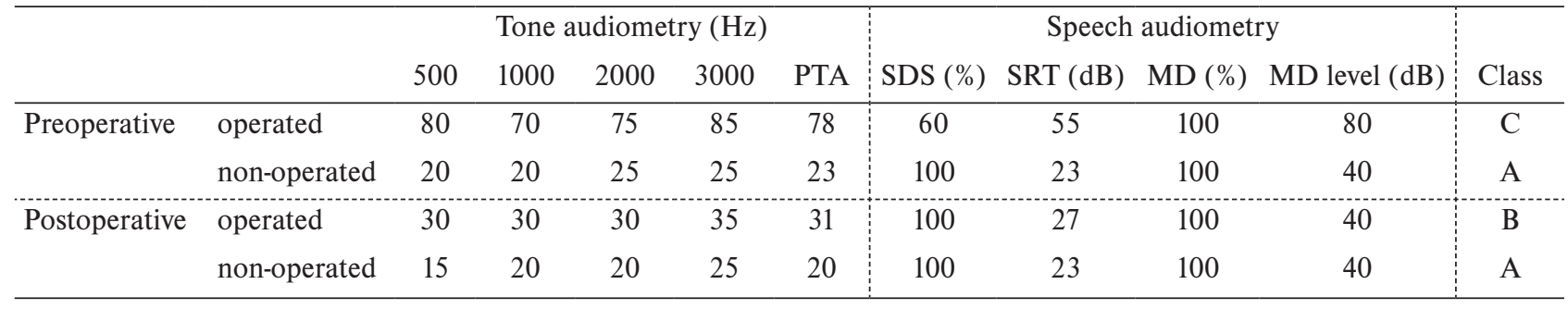

PTA - pure tone average $(\mathrm{dB})$ on $500 \mathrm{~Hz}-3 \mathrm{kHz}$, SDS - speech discrimination score, SRT - speech reception treshold, MD - maximal discrimination, MDlevel - level of maximal discrimination.

Abstracts were screened for relevance based on predetermined inclusion criteria and exclusion criteria. Duplicates were removed.

\section{Inclusion crietria}

1) complete journal articles

2) vestibular schwannoma (acoustic neuroma) only tumor type

3) surgical treatment regardless surgical approach

Exclusion criteria

1) inadequate audiometric data

2) opinion, editorial, or review articles

3) non-English language

4) use of any kind of radiotherapy

All papers were read and their references exhaustively checked for additional papers not occurring in the original search. Papers with any result of hearing improvement after surgical therapy has been included in the study and the list of articles can be seen in the Table 2.

\section{CASE REPORT}

The 32-year-old patient has been observed at the Department of Otorhinolaryngology and Head and Neck Surgery, 1st Faculty of Medicine, Faculty Hospital Motol since 2014 for small intracanalicular VS in the left ear with a size of $9 \times 5 \times 3 \mathrm{~mm}$, grade I according to the Koos classification (Fig. 1a). The patient had experienced 3 months of hearing loss with tinnitus and headaches around the left ear, without imbalance problems. This was the patient's reason for seeing a physician. Apart from hearing loss, further clinical examination did not show any pathology. Control magnetic resonance imaging half a year later showed that the tumour was stationary, as were the patient's symptoms. Subsequently, the patient appeared in the outpatient office 3 years later due to impaired hearing on the left side. The control MRI showed a growth progression of $+2 \mathrm{~mm}$ to $11 \times 7 \times 5 \mathrm{~mm}$. At this point the tumour was grade II according to the Koos classification (Fig. 1b). Audiologically, the patient presented useless hearing Class $\mathrm{C}$ according to AAO-HNS guidelines (PTA 78 dB measured on 500, 1000, 2000, 3000, 4000 $\mathrm{Hz}$; SDS 60\%; measured 5 days before the procedure) with elicitable otoacoustic emissions (DPOAE) on both sides (Fig. 2a). The thresholds for the individual frequencies of both ears and speech audiometry are shown clearly (Table 1). Electronystagmography showed no disorder. Subjectively, the patient was no longer able to take phone calls with the affected ear. Furthermore, he reported tinnitus, paroxysmal headaches and no imbalance issues. After discussing all therapeutic options, taking into account the
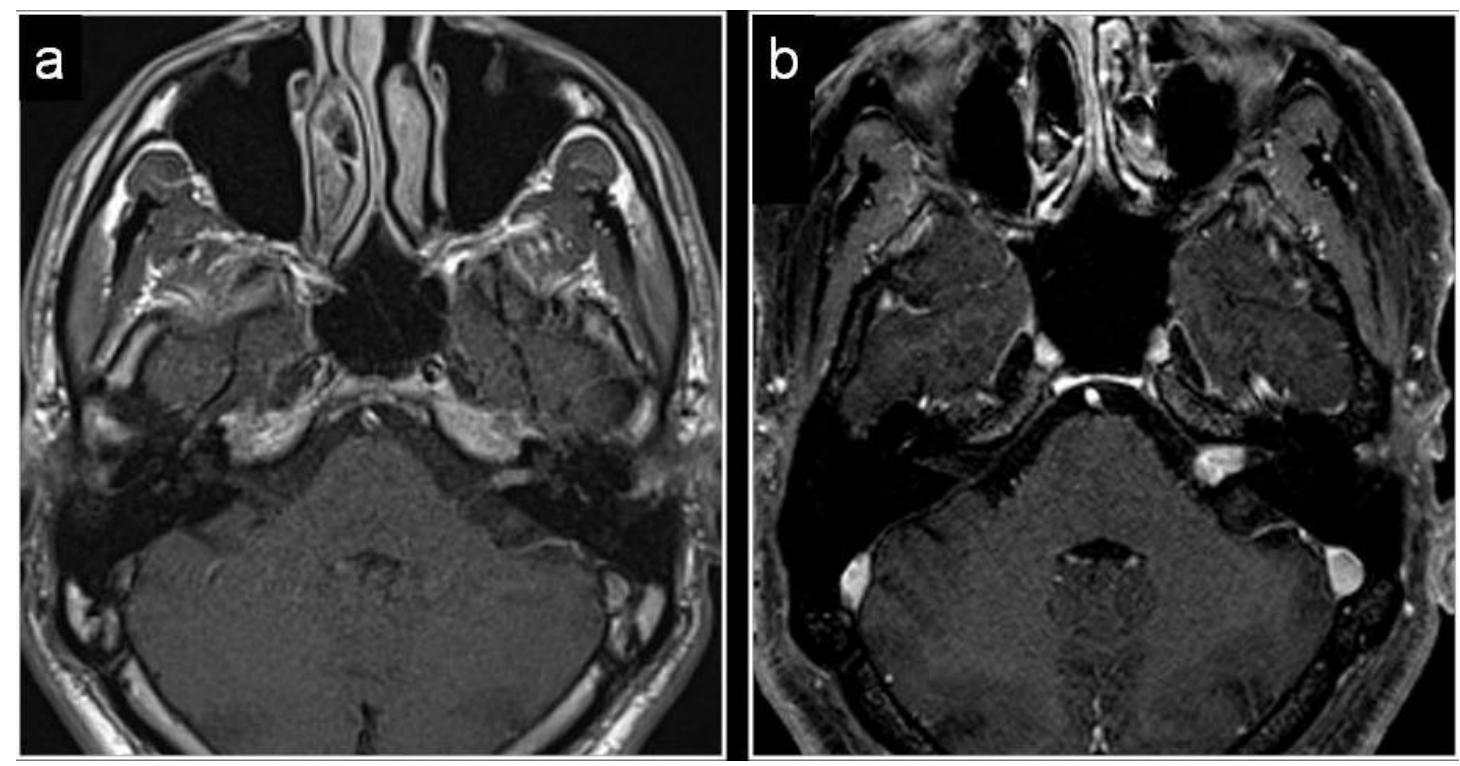

Fig. 1. Preoperative MR - progression of vestibular schwannoma on the left side: a) year 2014; b) year 2018. 

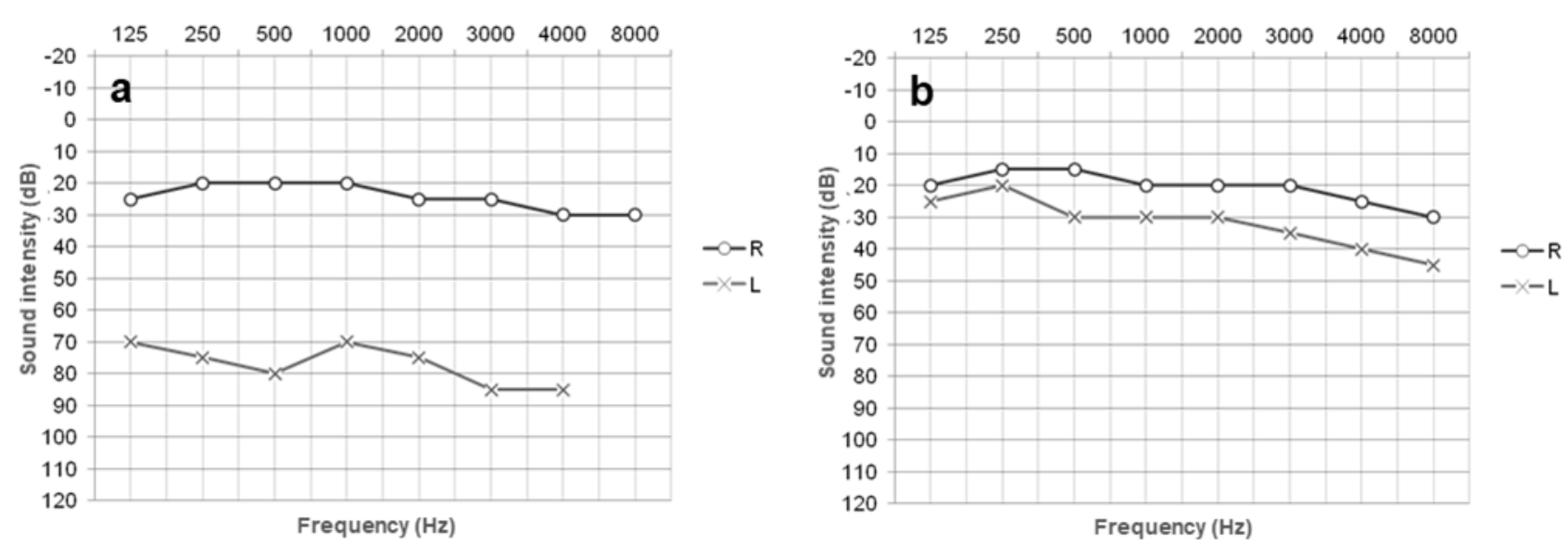

Fig. 2. Audiogram: a) 5 days prior the surgery: L - operated ear; b) 3.5 months after surgery: L - operated ear.

activity of the tumour, the patient's age and his personal preference, the surgical solution was chosen.

In 2018, the patient (36-year-old) underwent extirpation of the VS via retrosigmoid suboccipital craniotomy by opening of the internal auditory canal. Perioperative monitoring of evoked auditory potentials was not used due to preoperative useless hearing. After the exposure of the cerebellopontine angle, a small, extracanalicularly propagating tumour of typical appearance was visible. This allowed immediate identification of the facial nerve and vestibulocochlear nerve. To allow better space for preparation, we initially opened the internal acoustic meatus deep into the fundus and began to remove the tumour from the meatus. The preparation was challenging, especially in the case of the facial nerve, which ran directly ventrally from the tumour and was widespread in the area of the entrance to the internal auditory canal. The tumour was radically removed while maintaining a good stimulatory response of the facial nerve and intact auditory nerve. Both portions of the vestibular nerve were discontinued, the tumour arose from the supeior vestibular nerve (Fig. 3). The duration of the procedure from incision to suture was $5 \mathrm{~h}$.

The postoperative course was uneventful, without complications. The facial nerve function has been intact from the first postoperative day (1st degree according to House-Brackmann), only a slight decrease in active motor units was described during electromyography, tinnitus disappeared, vestibular compensation was reached quickly.

Wound healing was normal. Shortly after the operation, the patient stated that he was again able to make a telephone call with his operated ear. Audiologically, one week after the procedure, the PTA on the operated ear was measured at $43 \mathrm{~dB}$, speech audiometry is not routinely performed immediately after the procedure.

Three and a half months after the surgery, the average hearing threshold was $31 \mathrm{~dB}$, SDS $100 \%$ (Fig. 2b, Table 1), DPOAE were elicitable. Thus, according to AAO-HNS, hearing improved to a useful level (Class B). The only dominant complaint was neuralgic headaches at the wound site.

No difference was found when comparing the examination of stem evoked potentials before and after surgery. Bilateral supra-threshold responses with latencies within

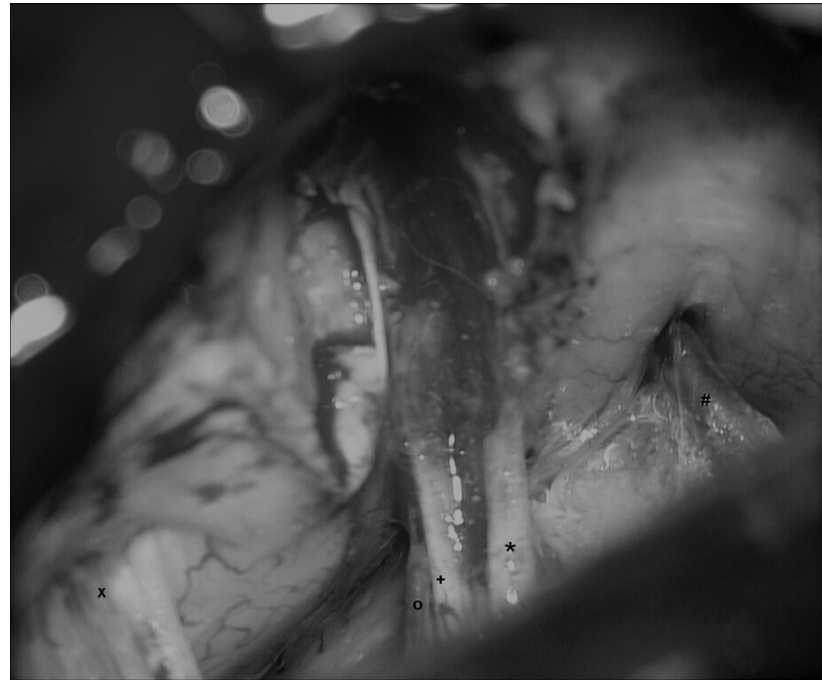

Fig. 3. Situation in the cerebellopontine angle and internal meatus at the end of the surgery: $\mathrm{x}$ - lower cranial nerves, $\mathrm{o}$ - labyrinthine artery, + - cochlear nerve, * - facial nerve, \# - superior petrosal vein.

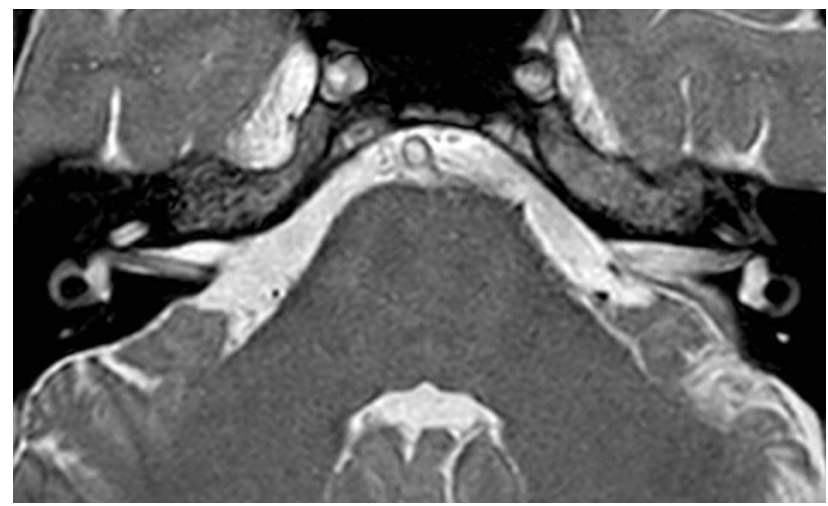

Fig. 4. Postoperative MRI.

the population norm, without interaural difference, were described bilaterally. No retrocochlear disorder was demonstrated.

On routinely performed magnetic resonance imaging 3 months after the surgery we saw a common postoperative condition, without signs of recurrence, with preservation of the nerve structures of the internal auditory canal (Fig. 4). 
A comparison of postoperative questionnaires revealed only minimal changes in the subjective perception of hearing impairment (Hearing handicap inventory): $4 / 100$ preoperatively versus $0 / 100$ postoperatively. No significant difference was found before and after surgery in the other questionnaires examining the individual symptoms, with the exception of the perception of headache (Headache handicap inventory). According to the patient, there was a distinct deterioration: $36 / 100$ vs 50/100 in this questionnaire. Bothersome headaches, however, were already present before surgery. A comparison of the questionnaires monitoring the overall condition of the patient (PANQUOL - Penn Acoustic Neuroma Quality of Life Scale + QoL36 - Quality of Life 36 questions) showed a significant deterioration of the score in the postoperative period: PANQUOL 35/130 vs 56/130; QoL 101/121 vs $80 / 121$.

\section{DISCUSSION}

There is a dearth of literature on hearing improvement following vestibular schwannanoma surgery (Table 2). Unfortunately, there are large discrepancies in the definition of hearing improvement, as well as in the description of possible prognostic factors. It is noteworthy that the majority of the articles are from the $80 \mathrm{~s}^{6}$ and $90 \mathrm{~s}^{6}$, with the numbers falling sharply thereafter. From this it can be concluded that hearing improvement after VS surgery remains rare.

At the current level of knowledge it is not possible to reliably determine the factors that may affect the improvement of hearing after VS surgery. Generally, the crucial goal of the surgeon should be auditory nerve decompression and revascularization of the vessels to the labyrinth ${ }^{2}$. In addition to being a technically challenging procedure, there is still a lack of indication criteria for such an attempt.

Currently, one of the most discussed parameters which could predict hearing improvement after surgery is a short time interval between hearing loss and time of surgery. Several authors described patients who experienced sudden loss of hearing up to 3 months prior to surgery and who's hearing improved after the procedure (Table 2). In our patient, hearing deterioration occurred in the longer period before surgery and did not present signs of sudden worsening.

Nowadays, otoacoustic emissions (OEA) are being analyzed for prognostic information regarding the residual hearing capacity of patients with VS. However, only a few reviewed papers discussed OEA, as this examination method has only become widespread during the past two decades. In any case, OEA were positive in the majority of improved patients, ours included (Table 2) (ref. ${ }^{6}$ ).

Interestingly, an uncommonly discussed factor related to hearing preservation probability was lower electronystagmography (ENG) function on the affected side, meaning that tumoural involvement of the superior vestibular nerve can be a positive prognostic factor for such attempts ${ }^{2,7,8}$. This finding is in accordance with the pre- sented case report, in which the tumour arose from the superior vestibular nerve, however it has not been proven on electronystagmography. Conversely, Inuoue et al. presented results of hearing improvement in patients with tumours originating from the inferior vestibular nerve ${ }^{6}$. Shelton and House did not find any correlation with ENG status ${ }^{9}$.

The definition of hearing improvement is another controversial topic. Nadol et al. postulated hearing improvement as greater than $15 \%$ in SDS and no greater than $15 \mathrm{~dB}$ in SRT (ref. ${ }^{14}$ ). Shelton and House presented stricter criteria for hearing improvement after VS resection - with improvement being greater than $20 \%$ in SDS and/or greater than $15 \mathrm{~dB}$ in SRT (ref. ${ }^{9}$ ). We believe the latter definition better represents the real impact on the patient. Furthermore, SRT is sometimes used equivalently to PTA, however, PTA is favored nowadays. Of note, it is believed that the contrariety between PTA and SRT is useful in the detection of malingerers ${ }^{24}$. In our case, there is a discrepancy in preoperative SRT and PTA, however this still fits with the observed improvement from Class C to B for both variables.

Another discrepancy can be found in PTA counting, because a $3 \mathrm{kHz}$ measurement (recommended by AAOHNS) is not always performed in published reports ${ }^{24}$. In our audiological assessments we count PTA starting at five frequencies, adding $4 \mathrm{kHz}$ to produce more significant results. We note that it is possible to detect patients in the papers discussed who met the criteria established by Shelton and House (Table 2).

The question remains of whether it will be possible to achieve these results routinely using currently available equipment and pharmacological support, as the auditory nerve is much more prone to damage if manipulated when compared to the facial nerve ${ }^{27}$. Useful technical notes of cochlear nerve preservation have been described in the pioneering work „Hearing preservation age“ by Jannetta et al. ${ }^{28}$.

We must also not forget the negative effects of postoperative hearing preservation. Several reports mention a higher risk of persistent tinnitus, especially if the tinnitus is perceived by the patient as bothersome ${ }^{29}$. We also know that in an effort to preserve the auditory nerve and the delicate structures of the inner ear, the surgeon is sometimes pushed towards a less radical tumour removal, especially in the fundus of the internal auditory canal, and patients are thus more exposed to the risk of a small tumour residue remaining behind ${ }^{30}$. In contrast, non-radical operation can be the surgeon's strategy when attempting to preserve cranial nerve function during vestibular schwannoma surgery ${ }^{16,26}$.

Last but not least, the question remains as to what role hearing preservation plays in the patient's overall perception of quality of life after surgery. Quality of life is slowly being included in management decisions in some centers $^{31}$. Although no conclusions can be drawn from a single-patient questionnaire study, it can be predicted that hearing preservation will not be a priority for some patients $^{32}$. Therefore, selection of such patients can be important in the choice of treatment strategy, both for one 


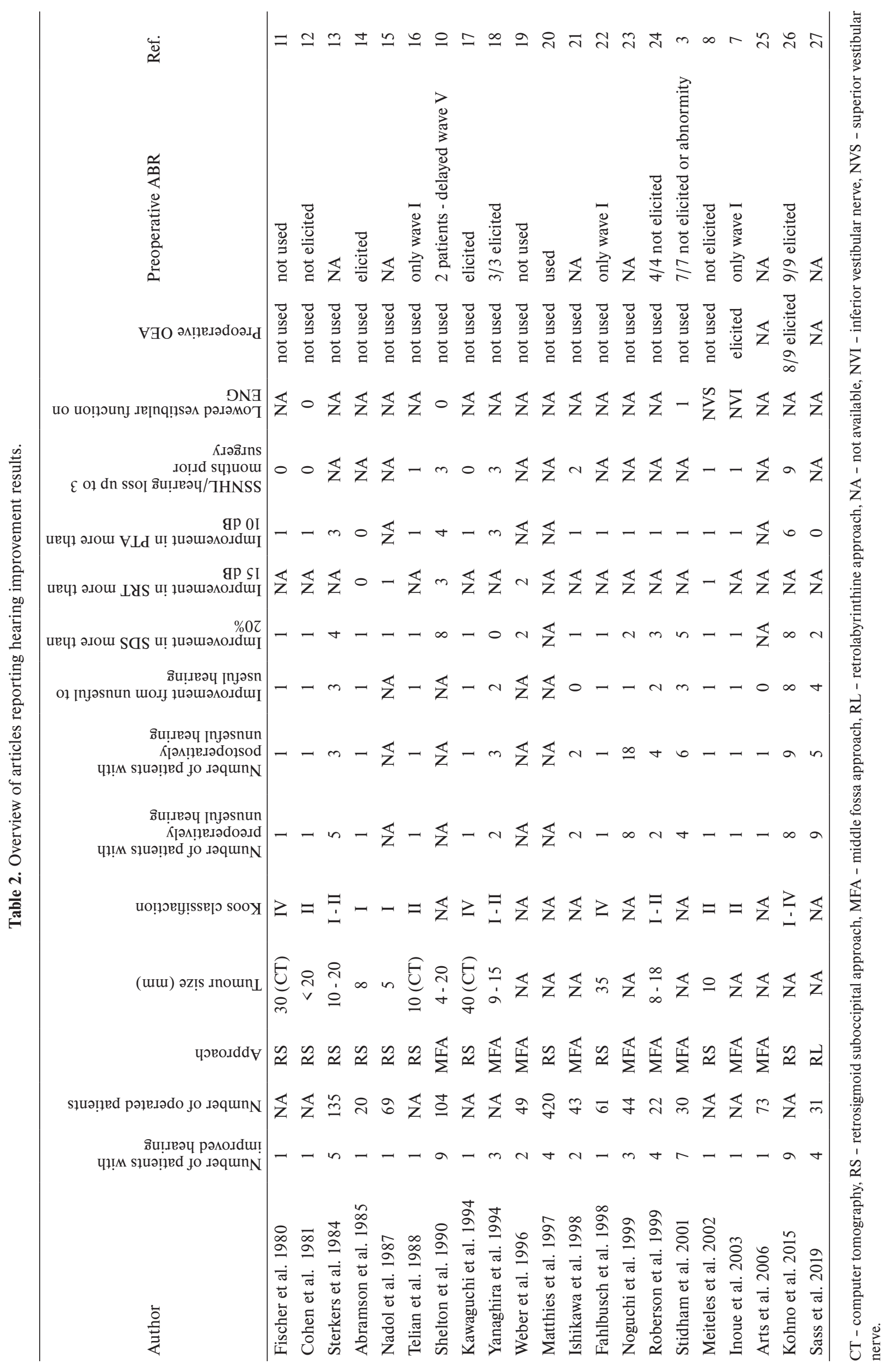


of the three basic treatment modalities and for the choice of surgical approach.

From this perspective, the experience, preferences and overall philosophy towards VS surgery at individual departments come to the fore.

\section{CONCLUSION}

The presented case of VS surgery by retrosigmoid suboccipital craniotomy describes the improvement of hearing from useless to useful levels. It somewhat justifies the effort to preserve hearing in selected patients with useless hearing, good cochlear function and small tumour size despite the widespread hearing preservation rule. Sudden sensorineural hearing loss prior to surgery, elicitable otoacoustic emissions and the origin from the superior vestibular nerve (proven on ENG) seem to be useful guides for predicting these results. However, the importance of postoperative hearing quality compared to other symptoms and complications remains debatable.

Acknowledgement: We thank Erick Harr for proofreading. Author contributions: ZF: surgery, manuscript writing; JL: questionnaire study; SH: audiometry, ABR analysis; ZC: vestibular follow-up; EZ: surgery; JB: surgery.

Conflict of interest statement: The authors state that there are no conflicts of interest regarding the publication of this article.

\section{REFERENCES}

1. Betka J, Chovanec M, Zverina E, Profant O, Lukes P, Skrivan J, Kluh J, Fik Z. 2011. Minimally Invasive Endoscopic and Endoscopy-Assisted Microsurgery of Vestibular Schwannoma. In: Iancu PC, editor. Advances in Endoscopic Surgery: InTech. p 191-216

2. Stidham KR, Roberson JB, Jr. Hearing improvement after middle fossa resection of vestibular schwannoma. Otol Neurotol 2001;22(6):91721.

3. Babbage MJ, Feldman MB, O'Beirne GA, Macfarlane MR, Bird PA. Patterns of hearing loss following retrosigmoid excision of unilateral vestibular schwannoma. J Neurol Surg B Skull Base 2013;74(3):16675 .

4. Sughrue ME, Yang I, Aranda D, Kane AJ, Parsa AT. Hearing preservation rates after microsurgical resection of vestibular schwannoma. J Clin Neurosci 2010;17(9):1126-9.

5. Walsh RM, Bath AP, Bance ML, Keller A, Rutka JA. Consequences to hearing during the conservative management of vestibular schwannomas. Laryngoscope 2000;110(2 Pt 1):250-5.

6. Inoue Y, Ogawa K, Kanzaki J. Hearing improvement after tumor removal in a vestibular schwannoma patient with severe hearing loss. Eur Arch Otorhinolaryngol 2003;260(9):487-9.

7. Meiteles LZ, Liu JK, Couldwell WT. Hearing restoration after resection of an intracanalicular vestibular schwannoma: a role for emergency surgery? Case report and review of the literature. J Neurosurg 2002;96(4):796-800.

8. Borgmann $\mathrm{H}$, Lenarz T, Lenarz M. Preoperative prediction of vestibular schwannoma's nerve of origin with posturography and electronystagmography. Acta Otolaryngol 2011;131(5):498-503.

9. Shelton C, House WF. Hearing improvement after acoustic tumor removal. Otolaryngol Head Neck Surg 1990;103(6):963-5.

10. Fischer G, Costantini JL, Mercier P. Improvement of hearing after microsurgical removal of acoustic neurinoma. Neurosurgery 1980;7(2):154-9.
11. Cohen NL, Ransohoff J, Jacobs J. Restoration of speech discrimination following suboccipital, transmeatal excision of extracanalicular acoustic neuroma. Otolaryngol Head Neck Surg 1985;93(1):126-31.

12. Sterkers JM, Sterkers O, Maudelonde C, Corlieu P. Preservation of hearing by the retrosigmoid approach in acoustic neuroma surgery. Adv Otorhinolaryngol 1984;34:187-92.

13. Abramson M, Stein BM, Pedley TA, Emerson RG, Wazen JJ. Intraoperative BAER monitoring and hearing preservation in the treatment of acoustic neuromas. Laryngoscope 1985;95(11):131822.

14. Nadol JB, Jr., Levine R, Ojemann RG, Martuza RL, Montgomery WW, de Sandoval PK. Preservation of hearing in surgical removal of acoustic neuromas of the internal auditory canal and cerebellar pontine angle. Laryngoscope 1987;97(11):1287-94.

15. Telian SA, Kemink JL, Kileny P. Hearing recovery following suboccipital excision of acoustic neuroma. Arch Otolaryngol Head Neck Surg 1988;114(1):85-7.

16. Kawaguchi T, Tanaka R, Kameyama S, Yamazaki H. Full recovery from deafness after removal of a large acoustic neurinoma associated with neurofibromatosis 2: case report. Surg Neurol 1994;42(4):326-9.

17. Yanagihara N, Murakami S, Asai M, Hatakeyama T, Zenke K. Hearing improvement following removal of acoustic neuroma with preoperative sudden deafness. Ear Nose Throat J 1994;73(12):886, 890-4.

18. Weber PC, Gantz BJ. Results and complications from acoustic neuroma excision via middle cranial fossa approach. Am J Otol 1996;17(4):669-75.

19. Matthies C, Samii M. Management of vestibular schwannomas (acoustic neuromas): the value of neurophysiology for evaluation and prediction of auditory function in 420 cases. Neurosurgery 1997;40(5):919-29; discussion 929-30.

20. Ishikawa K, Yasui N, Mineura K, Sasajima H, Togawa K. Significance of hearing preservation in acoustic neuroma surgery. Acta Otolaryngol Suppl 1998;537:16-9.

21. Fahlbusch $R$, Neu $M$, Strauss $C$. Preservation of hearing in large acoustic neurinomas following removal via suboccipito-lateral approach. Acta Neurochir (Wien) 1998;140(8):771-7; discussion 778.

22. Noguchi Y, Komatsuzaki A, Nishida H. Cochlear microphonics for hearing preservation in vestibular schwannoma surgery. Laryngoscope 1999;109(12):1982-7.

23. Roberson JB, Jr., Jackson LE, McAuley JR. Acoustic neuroma surgery: absent auditory brainstem response does not contraindicate attempted hearing preservation. Laryngoscope 1999;109(6):904-10.

24. Arts HA, Telian SA, El-Kashlan H, Thompson BG. Hearing preservation and facial nerve outcomes in vestibular schwannoma surgery: results using the middle cranial fossa approach. Otol Neurotol 2006;27(2):234-41.

25. Kohno $M$, Sora $S$, Sato $H$, Shinogami $M$, Yoneyama $H$. Clinical features of vestibular schwannomas in patients who experience hearing improvement after surgery. Neurosurg Rev 2015;38(2):331-41; discussion 341.

26. Sass HCR, Miyazaki H, West N, Hansen S, Moller MN, Caye-Thomasen P. Extended Retrolabyrinthine Approach: Results of Hearing Preservation Surgery Using a New System for Continuous Near Real-time Neuromonitoring in Patients With Growing Vestibular Schwannomas. Otol Neurotol 2019;40(5S Suppl 1):S72-S79.

27. Samii M, Turel KE, Penkert G. Management of seventh and eighth nerve involvement by cerebellopontine angle tumors. Clin Neurosurg 1985;32:242-72.

28. Jannetta PJ, Moller AR, Moller MB. Technique of hearing preservation in small acoustic neuromas. Ann Surg 1984;200(4):513-23.

29. Chovanec M, Zverina E, Profant O, Balogova Z, Kluh J, Syka J, Lisy J, Merunka I, Skrivan J, Betka J. Does attempt at hearing preservation microsurgery of vestibular schwannoma affect postoperative tinnitus? Biomed Res Int 2015;2015:783169.

30. Mazzoni A, Calabrese V, Moschini L. Residual and recurrent acoustic neuroma in hearing preservation procedures: neuroradiologic and surgical findings. Skull Base Surg 1996;6(2):105-12.

31. Sandooram D, Hornigold R, Grunfeld B, Thomas N, Kitchen ND, Gleeson M. The Effect of Observation versus Microsurgical Excision on Quality of Life in Unilateral Vestibular Schwannoma: A Prospective Study. Skull Base 2010;20(1):47-54.

32. Iyer AP, Gunn R, Sillars H. Quality of life after vestibular schwannoma surgery: does hearing preservation make a difference? J Laryngol Otol 2010;124(4):370-3. 